\title{
Structural Credit Modeling Under Stochastic Volatility
}

Marcos Escobar (Corresponding author)

Department of Mathematics, Ryerson University

Toronto M3A 2K7, Canada

Tel: 1-416-979-5000 E-mail: escobar@ryerson.ca

Tim Friederich

Department of Mathematics, Technische Universität München

Parkring 1185748 Garching b. Mnchen, Germany

Tel: 49-89-289-17417Ｅ-mail: friederich@tum.de

Mikhail Krayzler

Department of Mathematics, Technische Universität München

Parkring 1185748 Garching b. Mnchen, Germany

Tel: 49-89-289-17417Ｅ-mail: krayzler@tum.de

Luis Seco

Department of Mathematics, University of Toronto

Toronto M5S 2E4, Canada

Tel: 1-416-978-3984 E-mail: seco@math.utoronto.ca

Rudi Zagst

Department of Mathematics, Technische Universität München

Parkring 1185748 Garching b. Mnchen, Germany

Tel: 49-89-289-17401Ｅ-mail: zagst@tum.de

Received: February 16, 2012 Accepted: March 5, 2012 Published: May 1, 2012

doi:10.5539/ijsp.v1n1p20 URL: http://dx.doi.org/10.5539/ijsp.v1n1p20

\begin{abstract}
This paper presents a structural credit model with underlying stochastic volatility, a CIR process, combining the Black/Cox framework with the Heston Model. We allow to calibrate a Heston Model for a non-observable process as underlying of the Black/Cox Model. A closed-form solution for the price of a down-and-out call option on the assets with the debt as barrier and strike price is derived using the concept of optional sampling. Furthermore, estimators are derived with the Method of Moments for Hidden Markov Chains. As an application in Statistical Finance, the default probabilities of Merrill Lynch during the financial crisis are examined.
\end{abstract}

Keywords: Credit models, Barrier options, Stochastic volatility, Black/Cox model, Heston model

\section{Introduction}

In order to describe the performance of a company on a daily basis, we can often refer to quoted stock prices only. These prices reflect the market value of the company's equity. Yet, it's mainly the assets and the liabilities which drive the performance of a stock, and which may develop independently. The higher the leverage ratio (i. e. the ratio between debt and equity capital) the more important it becomes to consider these underlying factors. Yet in general, we cannot observe daily time series for assets or liabilities.

Following the theory of structural models, whose foundations have been laid mainly by Merton (1973) and Black and Cox (1976), the company's value i.e. its equity's value is modeled as an option on the asset value with the value of the debt 
as strike price. In this paper, the value is modeled as the value of a down-and-out call option (DOC) with the assets as underlying and the value of the debt as both, knock-out barrier and strike price. A simple call option pays the difference between the underlying and the strike price at maturity if this difference is positive. A down-and-out call option only pays this difference if the underlying does not fall below a certain threshold at any time before maturity. This threshold is called knock-out barrier. Knock-out barrier and strike price do not necessarily have to be equal (see Escobar et al., 2012).

Modeling the assets as a Brownian motion as in the Black/Cox Model would have the drawback of assuming normally distributed returns and constant volatility. However, in particular the financial crisis has shown that volatility is not constant over time. Heston Model, which is used here to model the assets allows the company's assets to have stochastic volatility. This model is chosen for its suitability in the pricing of financial products as well as for its closed-form expression for higher conditional moments. It should be noted that Heston Model is a continuous-time stochastic volatility (SV) process as oppose to popular discrete-time SV processes known as ARCH/GARCH (see Bollerslev, 1986). There is a recent literature on continuous-time extensions of GARCH models, see for example COGARCH by Brockwell et al., (2006); but the complexity of these extensions for estimation and pricing purposes makes well-established SV models as Heston model still dominant for academics and practitioners alike. The first part of the paper focuses on the modeling and introduces a closed-form formula for the down-and-out option. In the second part, estimators are derived using the method of moments on a discretization of the process as well as the mixing properties of the Hidden Markov Chain. This generalization is inspired by the work of Genon-Catalot et al., (2000) and allows for the calibration of the parameters of the asset process.

The model not only allows to describe the value of the company and its assets. It also enables to simulate multiple scenarios of possible paths how the stock would evolve over time according to the model. These scenarios can be analyzed under different aspects. As an example, the default probabilities in these scenarios are examined. In this example, the model is applied to a case which can be regarded as one of the key events of the financial crises: the takeover of Merrill Lynch by the Bank of America, which The Wall Street Journal headlined "The End of Wall Street".

This paper is organized as follows: Section 2.1 introduces the stochastic volatility model for the assets. Section 2.2 presents the relationship between assets and equity as well as some numerical results. Section 2.3 derives the estimation methodology, while in Section 3 the approach is applied to data from Merrill Lynch. Section 4 gives a conclusion of the study.

\section{The Stochastic Volatility Model}

Asset returns, and hedge fund returns in particular, are not normally distributed. Thus, from today's point of view, a Black/Scholes Model is barely able to map reality in an appropriate way, as one of the main assumptions is that the returns are normally distributed. Furthermore, the Black/Scholes Model assumes the volatility of the asset process to be constant over time. This characteristic feature is called homoscedasticity. However, even the stock market suggests that this is not the case. For example, it can be observed that those times when the stock markets take a hit, the (implied) volatility is higher than in "peaceful" times of strong markets, see e.g. Engle (1982) or Heston (1993). Therefore, we propose a model with stochastic volatility to describe the asset process which also implies a stochastic volatility model for the equity process of the company.

\subsection{Model Setup}

Let $(\Omega, \mathcal{F}, \mathbb{F}, \mathbb{Q})$ be a filtered probability space on the domain $\Omega$ with sigma algebra $\mathcal{F}$, filtration $\mathbb{F}=\left\{\mathcal{F}_{t}\right\}_{t \geq 0}$, and a probability measure $\mathbb{Q}$ on $(\Omega, \mathcal{F})$. The underlying asset process $A$ and the variance $v$ of that process can be expressed through the following SDEs:

$$
\begin{aligned}
d A(t) & =\mu A(t) d t+\sqrt{v(t)} A(t) d Z(t) \\
d v(t) & =\kappa_{v}\left(v_{\infty}-v(t)\right) d t+\varepsilon_{v} \sqrt{v(t)} d Z^{v}(t)
\end{aligned}
$$

where

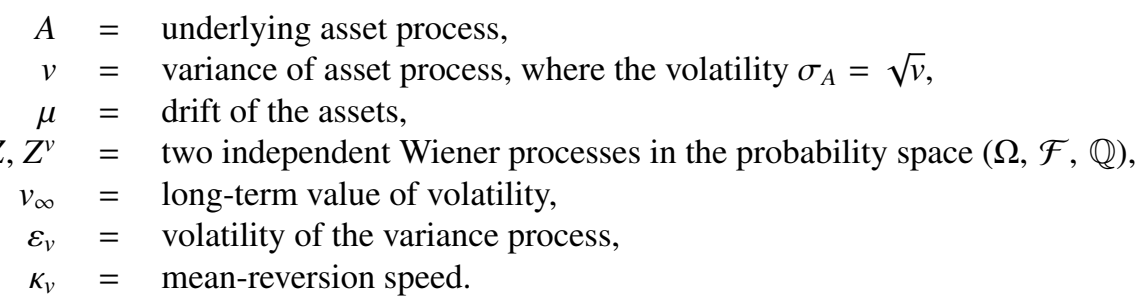

These assumptions provide the bare minimum to obtain a setting where closed-form solutions are available not only for the price of the credit products but also for a fast and reliable estimation of the parameters. For example, in a more general 
formulation, $Z$ and $Z^{v}$ can be correlated, however the assumption of zero correlation is required to obtain closed-form expressions for the estimators in Section 2.3. The parameters $v_{\infty}, \kappa_{v}$ and $\varepsilon_{v}$ have to fulfill the following conditions:

$$
\begin{aligned}
\kappa_{v} \cdot v_{\infty} & \geq \frac{1}{2} \varepsilon_{v}^{2} \\
\kappa_{v} & >0
\end{aligned}
$$

These conditions guarantee strict stationarity and $\alpha$-mixing, see Genon-Catalot et al. (2000) which is necessary for the derivation of estimators in Section 2.3. Furthermore, the barrier as the debt is exponentially growing with the risk-free rate $r$ :

$$
D(t)=D(0) \cdot e^{\int_{0}^{t} r d s}=D(T) \cdot e^{-\int_{t}^{T} r d s}
$$

where $D(t)$ denotes the value of the company's debt (or "liabilities") at time $t$.

The model defined by (1) and (2) is a so-called stochastic volatility model. Heston (1993) developed the model to overcome the shortcomings of the Black/Scholes Model where the latter seems to be too restrictive to map reality. Therefore, the model described by (1-2) is often referred to as the "Heston Model". In this model, the variance (2) is a stochastic process, introduced by Cox, Ingersoll, and Ross (1985), with the following features: First, the variance process has a long-term value or a "limit" $v_{\infty}$ around which it oscillates. Next, there is the mean-reversion speed $\kappa_{v}$. This parameter describes how fast the variance will adjust to its mean $v_{\infty}$. The term $\left(v_{\infty}-v(t)\right)$ is the deviation of the current variance from $v_{\infty}$. This term is weighted with the mean-reversion rate $\kappa_{v}$. Therefore, the higher $\kappa_{v}$ the faster the variance will tend towards $v_{\infty}$. Up until now, the variance process would be deterministic. In order to make it stochastic, the diffusion term $\varepsilon_{v} \sqrt{v(t)} d Z^{v}(t)$ is added, where $\varepsilon_{v}$ is the volatility of the variance process itself. The characteristics of the stochastic volatility model become "visible" in Figure 1.

\subsection{Barrier Options in the Stochastic Volatility Model}

The aim is to price a barrier option with strike $D(T)$ on the assets $A$ :

$$
C(t, A)=\mathbb{E}_{\mathbb{Q}}\left[e^{-\int_{t}^{T} r(s) d s} \cdot \max \{A(T)-D(T), 0\} \cdot \mathbf{1}_{\{\tau>T\}} \mid \mathcal{F}_{t}\right]
$$

where

$$
\begin{aligned}
C(t, A) & =\text { option price of the barrier option with underlying } A \text { at time } t, \\
\mathbf{1}_{\{\tau>T\}} & = \begin{cases}1 & \text { if } \tau>T \\
0 & \text { else }\end{cases}
\end{aligned}
$$

and $\tau$ is the time of default of the option, i.e. the first time, the asset process $A$ crosses the barrier $D$. It is modeled as a stopping time on the interval $(t, T]$ :

$$
\tau=\inf \left\{t^{\prime} \in(t, T]: A\left(t^{\prime}\right)<D\left(t^{\prime}\right)\right\}
$$

The symbol $\mathbb{E}_{\mathbb{Q}}$ denotes the expected value under the arbitrage-free measure $\mathbb{Q}$.

Equation (6) describes a down-and-out call option with underlying $A$, strike and knock-out barrier $D$. This means that when the price of the underlying hits or falls below the barrier the option expires (Note 1).

\subsubsection{Derivation of Option Pricing Formula}

For deriving the formula of the option price described by (6), the most straight-forward approach would be to solve the corresponding SDEs. However, in this particular case, the option price can also be derived with the help of optional sampling, which actually is more elegant than working through the SDEs. As it is shown in detail in the appendix, the price of the option is:

$$
C(t, A)=(A(t)-D(t)) \cdot \mathbf{1}_{\{\tau>t\}}
$$

The big advantage of (8) is that it provides a straight-forward inverse to calculate the asset value if both debt and equity value are known: Given the value of the equity $C(t)$ as well as the liabilities $D(t)$, and assuming that the company didn't default up to time $t$, i.e. $1_{\{\tau>t\}}$, the underlying assets $A(t)$ can be calculated as follows:

$$
A(t)=C(t)+D(t)
$$


Next, the option price - given a set of parameters specifying (1) and (2) - is calculated according to this formula and compared to a numerical simulation of the option price which is gained by evaluating (6). For this purpose, a large number of possible underlying asset paths is simulated with the same set of parameters. Then, the option price is the expected value of the discounted payoffs of all paths.

Figure 1 gives five examples for possible paths following the stochastic volatility model defined in (1) and (2). The parameters were chosen as follows, and are the same for all five paths:

$$
\begin{array}{lllll}
A(0) & \mathrm{r} & v(0)=v_{\infty} & \kappa_{v} & \varepsilon_{v} \\
100 & 0.04 & 0.01 & 0.5 & 0.1
\end{array}
$$

Figure 1 displays the variance process $v(t)$, the according volatility process which is the square root of the variance process, and the asset process $A(t)$, where the shading of lines in these three graphs indicates that the three time series belong together.

For validating the formula, the price for several options is calculated analytically according to (8). This price is then compared to a numerical simulation of the option price. For that purpose, 10000 paths under the given parameter set (the same as above, with $t=0$ and $T=5$ ) for the underlying asset process are simulated. For each of those paths, the payoff is calculated as

$$
P O=\max \{A(T)-D(T), 0\} \cdot \mathbf{1}_{\{\tau>T\}}
$$

in accordance with (6). The "numerical option price" is the discounted mean over those 10000 payoffs, i.e.

$$
C_{\text {num }}(t, A)=e^{-\int_{t}^{T} r(s) d s} \cdot \overline{P O}
$$

where $\overline{P O}=\frac{1}{n} \sum_{i=1}^{n} P O_{i}$.

Table 1 presents the outcomes of this test. From left to right, the columns give the values of the initial debt $D(t)=D(0)$, and the number of paths for which the payoff equals zero. Next, the discounted mean over all payoffs is provided $\left(C_{n u m}\right)$ along with the "correct" analytical option price, i.e. the option price according to Formula (8), together with their difference and the standard deviation of the 10000 values for $C_{\text {num }}$, i.e. the discounted payoff. The last four columns provide some validation for the derived formula in form of the confidence interval. Considering these number, this test numerically validates the analytical derivation of Equation (8).

\subsection{Estimation and Fitting of Parameters}

Having the formula for the barrier option at hand, the next tool required is the estimators for the method of moments. The aim is to find the parameters of the asset process which is calculated from the equity and debt time series employing (8). In the following, there are always three different time series of interest: (1) the underlying asset path $A$ of the company, (2) its debt $D$, which is also referred to as its liabilities, and (3) the company's equity $C$ which is also referred to as the stock price itself or the equity. Furthermore, there are different possible interpretations of the initial value of the debt $D(0)$. If $A(0)=1$, then $D(0)$ can be interpreted as the percentage of debt capital in the financing structure of the company at time $t=0$. If $C(0)=1$, then $D(0)$ is the leverage ratio the company operates with, because the leverage ratio is defined as the ratio between debt and equity capital.

The derivation of the estimators follows the lines of Genon-Catalot et al. (2000). As already mentioned, the two Wiener processes $Z$ and $Z^{v}$ denoting the stochastic component of the asset and volatility process are assumed to be uncorrelated. This leaves the following unknown parameters of the model described in (1) and (2): $\Theta=\left(\mu, v_{\infty}, \kappa_{v}, \varepsilon_{v}\right)$ which are to be fitted. The information on the debt $D$ as well as the risk-free rate $r$ can be observed and are assumed to be known. Actually, there is one more parameter which is unknown from the start which is $v(t)$, the level of variance at the time $t$ the option is to be priced for (usually, $t=0$ ). However, having $v_{0}=v(0)$ as well as $v_{\infty}$ as free parameters might bear problems for the fitting. Therefore, it is assumed that $v_{0}=v_{\infty}$. Note that this does not imply that $v_{\infty}$ is equal to the current volatility at the time the option price is calculated. As the volatility $v$ itself is not observable and thus cannot directly influence the fitting, $v_{\infty}$ and $v_{0}$ are rather regarded as two parameters of the model.

First of all, the actual calculation of suitable estimators requires some preparation. Assuming the model given by Equations (1) and (2), an application of Itô's lemma to the process $Y:=\log A$ immediately shows:

$$
d Y(t)=\left(\mu-\frac{v(t)}{2}\right) d t+\sqrt{v(t)} d Z(t)
$$


Under the condition that the variance process (2) is known and assuming $Y(0)=0$ i.e. $A(0)=1$,

$$
(Y(t) \mid v):=(Y(t) \mid v(s), 0<s<t) \sim \mathbf{N}\left(\mu \cdot t-\frac{1}{2} \int_{0}^{t} v(s) d s, \int_{0}^{t} v(s) d s\right)
$$

where $X \sim \mathbf{N}\left(\mu, \sigma^{2}\right)$ means that the random variable $X$ is normally distributed with mean $\mu$ and variance $\sigma^{2}$.

Lemma 2.1 Assume the partition $\left\{t_{i}\right\}_{i=1}^{n}$ of the time interval $[0, t]$ where $\Delta:=t_{i}-t_{i-1}$. Set

$$
\begin{aligned}
& R(i)=\left(\mu-\frac{\bar{V}(i)}{2}\right) \cdot \sqrt{\Delta}+\frac{1}{\sqrt{\Delta}} \int_{(i-1) \Delta}^{i \Delta} \sqrt{v(s)} d Z(s) \\
& \bar{V}(i)=\frac{1}{\triangle} \int_{(i-1) \Delta}^{i \Delta} v(s) d s
\end{aligned}
$$

then

$$
R(i)=\frac{Y(i \triangle)-Y((i-1) \triangle)}{\sqrt{\triangle}}
$$

Using (12) and the fact that the sum of two normally distributed random variables is also normally distributed, it follows that:

$$
(R(i) \mid v) \sim \mathbf{N}\left(\sqrt{\triangle} \mu-\frac{1}{2 \sqrt{\Delta}} \int_{(i-1) \Delta}^{i \Delta} v(s) d s, \frac{1}{\triangle} \int_{(i-1) \Delta}^{i \Delta} v(s) d s\right)
$$

and in terms of (13)

$$
(R(i), \mid \bar{V}) \sim \mathbf{N}\left(\left(\mu-\frac{1}{2} \bar{V}(i)\right) \cdot \sqrt{\triangle}, \bar{V}(i)\right) .
$$

The concept of $\alpha$-mixing was introduced by Rosenblatt (1956) under the term "strongly mixing". A formal definition of $\alpha$-mixing can be found in the appendix. It can be interpreted as a form of asymptotic independence of a process: $X(t)$ and $X(s)$ are more nearly independent the farther apart $s$ and $t$, as commented by Davidson (1993). Thus, $\alpha$-mixing is a weaker form of dependence. Under the mixing condition a central limit theorem can be shown to hold for general processes. A deeper discussion of $\alpha$-mixing and other forms of the mixing-condition can be found in Bradley (2005).

Proposition 2.2 Assume the process (2) satisfies (3) and (4). Then, for the process (14) there exist the following estimators for the moments of $R$ :

$$
\begin{array}{ccc}
\frac{1}{n} \sum_{i=0}^{n-1} R(i+1) & \stackrel{\text { a.s. }}{\longrightarrow} & \sqrt{\Delta} \mu-\frac{\sqrt{\Delta}}{2} \mathbb{E}[\bar{V}(1)] \\
\frac{1}{n-1} \sum_{i=0}^{n-2} R(i+1) R(i+2) & \stackrel{\text { a.s. }}{\longrightarrow} & \Delta \mu^{2}-\Delta \mu \mathbb{E}[\bar{V}(1)]+\frac{\Delta}{4} \mathbb{E}[\bar{V}(1) \bar{V}(2)] \\
\frac{1}{n} \sum_{i=0}^{n-1} R^{2}(i+1) & \stackrel{\text { a.s. }}{\longrightarrow} & \Delta \mu^{2}-(\Delta \mu-1) \mathbb{E}[\bar{V}(1)]+\frac{\Delta}{4} \mathbb{E}^{2}\left[\bar{V}^{2}(1)\right] \\
\frac{1}{n-1} \sum_{i=0}^{n-2} R^{2}(i+1) R(i+2) & \stackrel{\text { a.s. }}{\longrightarrow} & \left(\Delta \mu^{2}-(\Delta \mu-1) \mathbb{E}[\bar{V}(1)]+\frac{\Delta}{4} \mathbb{E}^{2}\left[\bar{V}^{2}(1)\right]\right) \\
\frac{1}{n} \sum_{i=0}^{n-1} R^{4}(i+1) & \stackrel{\text { a.s. }}{\longrightarrow} & \Delta^{2} \mu^{4}+\left(6 \Delta \mu^{2}-2 \Delta^{2} \mu^{3}\right) \mathbb{E}[\bar{V}(1)] \\
& & \left(\frac{3}{2} \Delta^{2} \mu^{2}-6 \Delta \mu+3\right) \mathbb{E}\left[\bar{V}^{2}(1)\right]
\end{array}
$$


where $\mathbb{E}[\bar{V}(1)], \mathbb{E}\left[\bar{V}^{2}(1)\right]$, and $\mathbb{E}[\bar{V}(1) \bar{V}(2)]$ can be calculated as (see Proposition 4.1 in Genon-Catalot et al., 2000):

$$
\begin{aligned}
\mathbb{E}[\bar{V}(1)] & =v_{\infty} \\
\mathbb{E}\left[\bar{V}^{2}(1)\right] & =v_{\infty}^{2}+\frac{\varepsilon_{v}^{2} v_{\infty}}{\kappa_{v}} \frac{\left(\kappa_{v} \Delta-1+e^{-\kappa_{v} \Delta}\right)}{\kappa_{v}^{2} \Delta^{2}} \\
\mathbb{E}[\bar{V}(1) \bar{V}(2)] & =v_{\infty}^{2}+\frac{\varepsilon_{v}^{2} v_{\infty}}{2 \kappa_{v}} \frac{\left(1-e^{-\kappa_{v} \Delta}\right)^{2}}{\kappa_{v}^{2} \Delta^{2}}
\end{aligned}
$$

In order to find the optimal parameters, the sum of deviations between the empirical and theoretical statistics of equations (16) to (20) is minimized:

$$
\min _{\Theta} \sum_{i \in\{(16),(17),(18),(19),(20)\}}\left(\operatorname{empStat}_{i}(\Theta)-\text { theoStat }_{i}(\Theta)\right)^{2}
$$

where empStat $t_{i}$ denotes the empirical estimator (i.e. the left hand side) of equation $i$, and theoS tat $t_{i}$ the theoretical estimator (i.e. the right hand side). (24) represents the basic optimization problem of how to fit the parameters. This minimization could be avoided if the system of equations (16-20) led to closed-form solutions, but the nonlinearity of the system avoids such a quick path. Equation (24) could be altered by assigning different weights on the specific estimators or completely neglecting one or more of the five estimators. Theoretically, all these approaches would lead to consistent estimators although not efficient ones unless using a suitable more complicated set of weights as in the Generalized Method of Moments. Furthermore, we could also include moments of order higher than 4. However, the theoretical estimators get more complex and contain the parameters with higher exponents. This would make the numerical estimations less stable.

\section{Application: Merrill Lynch and the Financial Crisis}

In order to show an application of the model, one of the most spectacular stories of the financial crises is scrutinized: the downfall of former Wall-Street giant Merrill Lynch (Note 2). Merrill Lynch stocks have long been deemed a save investment. But in September 2008, the existence of Merrill Lynch could only be saved by the takeover by the Bank of America. The stock price reached its peak in early 2007 being close to 100 USD. After still announcing record earnings in early 2007, Merrill Lynch was severely struck by the subprime crisis. In the summer of 2008, the events speeded up dramatically. Finally, the investment bank with the bull as figurehead fell prey to the bear. On September 15, Bank of America announced its intention to acquire Merrill Lynch. This way, Merrill Lynch was prevented from pursuing the same tragic fate as Lehman Brothers which were filing for insolvency that very same day.

The first period which is to be examined in this example is the time between July 2001 and June 2007. The performance over that time horizon seemed to be satisfying (see Figure 2), yet what was already striking is the fairly high volatility of the stock price. The daily mean of log-returns is $0.0227 \%$ (corresponding to an annual return of $5.71 \%$ which is calculated assuming 252 trading days per year), the standard deviation is $1.85 \%$ (which can be approximated by an annual standard deviation of $29.35 \%$ ), the skewness is -0.140 , and the kurtosis is 6.06 . But at first sight, there seemed to be no obvious sign that Merrill Lynch could default so soon.

\subsection{Fitting the Model Parameters}

The introduced stochastic volatility model requires several parameters which have to be determined before the model can be applied: the time to maturity $T$, the risk-free rate $r$, and the level of debt $D(0)$. The time to maturity is the time until the barrier option introduced in Section 2.2 extinguishes. It is therefore assumed to represent the average time to maturity of the company's liabilities. Unfortunately, this information is not provided by Merrill Lynch. For that reason, the time to maturity is assumed to be 5 years, i.e. $T=5$ to represent the average time of maturity of Merrill Lynch's liabilities. In order to get an appropriate risk-free rate, the average of the 5-year treasury rate over the according time horizon is used. Therefore, $r=3.93 \%$.

Furthermore, the initial debt $D(0)$ is required. In order to gain that information, the adjusted leverage ratio (Note 3 ) is extracted from the company's annual reports which are summarized in Table 2. The average adjusted leverage ratio over the considered time horizon is 12.7. However, note that Merrill Lynch defines the leverage as assets (not liabilities) divided by equity. Thus, the adjusted leverage ratio according to the common definition, is 11.7 . In order to define the initial value of the debt $D(0)$ assume that the initial value of the equity is $C(0)=1$. Therefore, $D(0)=11.7$.

The problem with fitting the model parameters is that the variance process cannot directly be observed, in particular $\kappa_{v}$ and $\varepsilon_{v}$. Thus, a sufficiently large number of data points is required in order to capture the characteristics and influence of these parameters. For finding the model parameters, the procedure works as follows: A value for $\varepsilon_{v}$ is fixed, and the 
remaining parameters $\mu, v_{\infty}$, and $\kappa_{v}$ are fitted. The optimal set of parameters (including the optimal $\varepsilon_{v}$ ) is that set which minimizes the error function (i.e. the sum of squared residuals as described in (24) as a global minimum.

The parameters $\Theta=\left(\mu, v_{\infty}, \kappa_{v} \varepsilon_{v}\right)$ for the time series of the Merrill Lynch stock between July 2001 and June 2007 are the following:

$\begin{array}{lllll}\mu & \mathrm{r} & \nu_{\infty} & \kappa_{v} & \varepsilon_{v} \\ 0.040916 & 0.000323 & 0.355731 & 0.5 & 0.012545\end{array}$

At first sight, the long-term variance seems to be fairly low. Yet, one has to bear in mind that the model has one important assumption which is that the debt is deterministic, i.e. it has no stochastic component and does not allow for a volatility of the returns of the debt. Thus, only the volatility of the equity can influence the volatility of the assets and vice versa. Furthermore, the leverage ratio is fairly high which dilutes the volatility of the equity in the asset process.

The value of $\kappa_{v}$ already indicates the necessity to apply the stochastic volatility model, as the parameter is clearly different from $0\left(\kappa_{v}=0\right.$, under Equation 4, would make the stochastic volatility model a common Black/Scholes Model). However, as this statement has no scientific relevance, an ARCH-test is applied. This test has been developed by Engle (1982) in order to test time series for homoscedasticity (constant volatility over time). In this case, the hypothesis of constant volatility can be rejected at a significance of $4.8 \cdot 10^{-7}$ emphasizing the use of the stochastic volatility model.

Figure 3 shows the histogram of daily returns of Merrill Lynch between July 2001 and June 2007. Furthermore, the normal distribution with same mean and standard deviation as the historic distritbuion as well as the distribution according to the stochastic volatility model is displayed. For the latter, more than 1 million daily asset returns ( 1000 time series each consisting of 1506 daily returns) have been simulated with the estimated parameters and the according equity returns have been calculated. The graph already shows that the stochastic volatility model maps reality much better than a common Black Scholes model. In order to validate this observation, a chi-square test is applied (see Chernoff and Lehmann, 1954). First, it is tested whether the historic returns follow a normal distribution. The test statistic with value 961.85 is clearly higher than the $95 \%$ critical value of the chi-square distribution 98.48 (accounting for two estimated parameters, mean and standard deviation). Therefore, the hypothesis can clearly be rejected. Next, the stochastic volatility model is tested. The resulting value of the test statistic is 89.82, and thus lower than the critical value of 96.22 (now accounting for four estimated parameters). As we cannot reject the hypothesis, we conclude that the stochastic volatility model maps the distribution of historic returns well.

\subsection{The Default Risk of Merrill Lynch}

One interesting aspect which is worth to study is the default risk of Merrill Lynch. In July 2007, all the information which has been used so far was known. Therefore, the estimated parameters $\Theta=\left(\mu, v_{\infty}, \kappa_{v}, \varepsilon_{v}\right)$ reflect the information given at that point of time. In order to study the default risk, 10000 underlying asset time series for Merrill Lynch are simulated over a 100-year time horizon applying the estimated parameters $\mu, v_{\infty}, \kappa_{v}$, and $\varepsilon_{v}$ starting on July 1, 2007. As already discussed above, it is arguable whether these simulated asset time series reflect Merrill Lynch's true assets. However, a model is always a simplification of reality, and despite the shortcomings of the simulated asset time series, this does not affect the validity of the equity time series gained by that model.

Every simulated asset time series is compared to the debt which is assumed to follow (5). Merrill Lynch would have to declare insolvency once its liabilities exceeded its assets. Therefore, the time of default is calculated as $\tau$ according to (7), as the first point of time where the assets fall below the debt. Figure 4 shows the results of that test: As already mentioned, 10000 asset time series have been simulated daily over a time horizon of 100 years. Figure 4 indicates how many paths default in the respective year, for example, 11 of them already default within the first year which represents July 2007 until June 2008, 34 default in the second year which represents July 2008 until June 2009. The last bar comprises those paths which have not defaulted within the first 100 years (i.e. until June 2107) which are 5913 paths. As the total of simulated time series is 10 000, the numbers can easily be interpreted as default probabilities in the respective years: The probability for a default amounts to $0.11 \%$ in the first year (starting on July 2007) and $0.34 \%$ in the second year.

With that information another interesting question can be answered: What is the probability for Merrill Lynch to survive over a certain span of time? This answer is given in Figure 5. Assuming the probabilities for the event of a default given in Figure 4, this figure provides the probability that Merrill Lynch survives until the respective year, which is 1 minus the sum over the default probabilities until that year. As the time series have been simulated daily, Figure 5 provides an almost continuous function. This graph tells that, for example, the probability to survive 10 years is $88.88 \%$, and the probability to survive 100 years is $59.13 \%$.

Figure 4 already points out that there is a significant default probability for Merrill Lynch in the years from July 2007 on, given the information described above. The probability for a default until June 2009 would be estimated to be $0.45 \%$ by 
this model. Although this number still seems to be fairly low (but is not negligible though), it can already be concluded that even Merrill Lynch bears a certain default risk. However, the takeover by the Bank of America did not mean a default for Merrill Lynch. Merrill Lynch accepted an offer by the Bank of America in order to overcome its own default risk, and the Bank of America finally paid 50 billion USD for that acquisition according to the Wall Street Journal.

\subsection{Development of Default Risk}

It is even more interesting to compare the findings so far with the condition of Merrill Lynch not before but in the middle of the financial crisis. Therefore, the situation of Merrill Lynch in January 2008 is examined. In order to have a comparable data basis, but which still allows to employ the stochastic volatility model, the time between January 2003 and December 2007 is considered for fitting the parameters. The average 5-year US treasury rate is 3.94\%, and from the average adjusted leverage ratio we get $D(0)=12.6$.

The fitted parameters $\Theta=\left(\mu, v_{\infty}, \kappa_{v}, \varepsilon_{v}\right)$ for Merrill Lynch based on the time between January 2003 and December 2007 are the following:

$\begin{array}{lllll}\mu & \mathrm{r} & \nu_{\infty} & \kappa_{v} & \varepsilon_{v} \\ 0.041350 & 0.000637 & 0.595501 & 0.5 & 0.024382\end{array}$

Once more, 10000 asset paths are simulated with those parameters and the event of default is examined. Figure 7 shows the number of defaults in the respective year. In this graph, the first year represents the time from January 2008 until December 2008, the second year is 2009, and so on. Especially when comparing these outcomes to those presented in Figure 4, the highly increased default risk becomes obvious. In the new setting, $0.47 \%$ of the simulated time series default within the first year (compared to $0.11 \%$ ), and $6.27 \%$ default within the first three years (compared to $1.40 \%$ ). In only $41.39 \%$ of the time series, the company does not default over the simulated time horizon of 100 years (compared to $59.13 \%$ ). Figure 8 displays the development of the survival probabilities for the two examined periods (the first year of a possible default starts in July 2007, or January 2008). The survival probability decreases notably. Especially in the first years, the probability to survive is dramatically lower for the situation in January 2008. This means that already on January 1st, 2008 this model only shows a 3:1 chance for Merrill Lynch to survive the next ten years. Therefore, the "unexpected" development of July, August and September 2008 does not really come as such a big surprise anymore. Considering that Merrill Lynch actually has not defaulted in September 2008 when being taken over by the Bank of America, it was almost clear that with these prospects, something "had to happen".

Furthermore, Figure 8 compares the survival probabilities of this model with the survival probabilities derived from the S\&P credit rating of Merrill Lynch. In January 2008, Merrill Lynch was rated "A+". Assuming that the transition matrix for the rating classes will not change over the next 100 years, yields the survival probabilities indicated by the dotted line. It becomes clear that, in particular, in the near future, the credit rating severely underestimates the probability of a default compared to the credit model under stochastic volatility.

\section{Conclusion}

This paper presented a model for a company's asset process if only the equity process is observable. Companies' equity is regarded as a barrier option, namely a down-and-out call on the company's assets where barrier and strike price are equal to the debt. The assets are modeled with stochastic volatility providing for more degrees of freedom than a common Black/Scholes Model. Thus, this paper combines the credit model as in Black and Cox (1976) with the stochastic volatility model in Heston (1993). As a first result, a closed-form solution for the price of a down-and-out call option on the assets with the debt as barrier and strike price has been derived. Second, a method of gaining consistent estimators was derived from Genon-Catalot et al. (2000), and five estimators were given explicitly which are utilized during the fitting process for single time series. Given the equity value of the company and information on the liabilities, we can derive an asset process where the assets have stochastic volatility. These contributions provide a computationally and statistically friendly setting for practitioners in economics and finance.

The applications in this study have been limited to one dimension. One potential extension of this research is to look at several companies and estimate their dependence structure within our stochastic volatility setting. Furthermore, it would also be of interest to examine a setting in the stochastic volatility model where the barrier is not equal to the strike price and/or where the debt is assumed to follow a stochastic process to account for uncertainty on the liability side as well. Yet, this would probably make it a lot more difficult to arrive at (closed-form) formulas for option prices or to obtain estimators with sound statistical properties. However, models with a higher complexity than the Black/Scholes Model where assets follow a Geometric Brownian Motion are very important, in particular stochastic volatility models which are able to better capture realistic features of market behavior, and thus better describe reality. 


\section{Appendix}

Derivation of Option Pricing Formula The following lemma is stated from Zagst (2002, Theorem 2.21, p. 21):

Lemma 5.3 (Optional Sampling) Let $(\Omega, \mathcal{F}, \mathbb{Q}, \mathbb{F})$ be a filtered probability space and $X=\left(X_{t}\right)_{t \geq 0}$ a right-continuous martingale which means that all paths of $X_{t}$ are right-continuous. Furthermore, let $\tau$ be a stopping time and $0 \leq s \leq t<$ $\infty$. Then, for all $t \geq 0$ it holds $\mathbb{Q}$-a.s. that

$$
\mathbb{E}\left[X_{t \wedge \tau} \mid \mathcal{F}_{s}\right]=X_{s \wedge \tau}
$$

where the stopped process $X_{t \wedge \tau}$ is defined as

$$
X_{t \wedge \tau}(\omega):=\left\{\begin{array}{lll}
X_{t}(\omega) & \text { if } t \leq \tau(\omega) \\
X_{\tau}(\omega) & \text { if } t>\tau(\omega)
\end{array}\right.
$$

In the present scenario, the option (6) can be rewritten as

$$
\begin{aligned}
C(t, A) & =\mathbb{E}_{\mathbb{Q}}\left[e^{-\int_{t}^{T} r(s) d s} \cdot \max \{A(T)-D(T), 0\} \cdot \mathbf{1}_{\{\tau>T\}} \mid \mathcal{F}_{t}\right] \\
& =\mathbb{E}_{\mathbb{Q}}\left[e^{-\int_{t}^{T} r(s) d s} \cdot(A(T)-D(T)) \cdot \mathbf{1}_{\{\tau>T\}} \mid \mathcal{F}_{t}\right]
\end{aligned}
$$

because if the asset value is below the value of the debt at maturity, i.e. $A(T)-D(T)<0$, the indicator function $\mathbf{1}_{\{\tau>T\}}$ with $\tau=\inf \left\{t^{\prime}: A\left(t^{\prime}\right)<D\left(t^{\prime}\right)\right\}$ already is zero. Thus, taking the maximum becomes redundant.

Now define

$$
X_{t}=X(t):=\tilde{A}(t)-\tilde{D}(t)
$$

with the discounted processes

$$
\begin{aligned}
& \tilde{A}(t) \quad:=e^{-\int_{0}^{t} r(s) d s} A(t) \\
& \tilde{D}(t) \quad:=e^{-\int_{0}^{t} r(s) d s} D(t)
\end{aligned}
$$

for assets and debt. As $A(t)$ and $D(t)$ are continuous $X_{t}$ is continuous, and thus, $X_{t \wedge \tau}$ is continuous as well, in particular for $t=\tau$. As a tradable underlying $\tilde{A}(t)$ can be assumed a $\mathbb{Q}$-martingale. Therefore, $X_{t}$ is a martingale. The stopped process is

$$
\begin{aligned}
X_{t \wedge \tau}(\omega) & = \begin{cases}\tilde{A}(t)-\tilde{D}(t) & \text { if } t \leq \tau(\omega) \\
0 & \text { if } t>\tau(\omega)\end{cases} \\
& =(\tilde{A}(t)-\tilde{D}(t)) \cdot \mathbf{1}_{\{\tau>t\}}
\end{aligned}
$$

Using the stopped process, the option price (27) is:

$$
\begin{aligned}
C(t, A) & =\mathbb{E}_{\mathbb{Q}}\left[e^{-\int_{t}^{T} r(s) d s} \cdot(A(T)-D(T)) \cdot \mathbf{1}_{\{\tau>T\}} \mid \mathcal{F}_{t}\right] \\
& =e^{\int_{0}^{t} r(s) d s} \cdot \mathbb{E}_{\mathbb{Q}}\left[(\tilde{A}(T)-\tilde{D}(T)) \cdot \mathbf{1}_{\{\tau>T\}} \mid \mathcal{F}_{t}\right] \\
& =e^{\int_{0}^{t} r(s) d s} \cdot \mathbb{E}_{\mathbb{Q}}\left[X_{T \wedge \tau} \mid \mathcal{F}_{t}\right] \\
& =e^{\int_{0}^{t} r(s) d s} \cdot X_{t \wedge \tau} \\
& =e^{\int_{0}^{t} r(s) d s} \cdot(\tilde{A}(t)-\tilde{D}(t)) \cdot \mathbf{1}_{\{\tau>t\}} \\
& =(A(t)-D(t)) \cdot \mathbf{1}_{\{\tau>t\}}
\end{aligned}
$$

Given that the company didn't default up to time $t$, i.e. $\mathbf{1}_{\{\tau>t\}}=1$,

$$
C(t, A)=A(t)-D(t) .
$$

Definition 5.4 ( $\alpha$-mixing) Let $X$ be a stochastic process (which is a sequence of random variables $\left\{X_{k}\right\}_{k \in \mathbb{Z}}$ ) on a probability space $(\Omega, \mathcal{F}, \mathbb{Q})$ and define

$$
\mathcal{F}_{A}^{B}:=\sigma\left(X_{k}, k \in[A, B]\right)
$$


for $-\infty \leq A \leq B \leq \infty . \sigma(E)$ denotes the generated $\sigma$-algebra with generator $E$.

Furthermore,

$$
\alpha(n):=\sup _{j \in \mathbb{Z}} \alpha\left(\mathcal{F}_{-\infty}^{j}, \mathcal{F}_{j+n}^{\infty}\right) .
$$

with

$$
\alpha\left(\mathcal{F}_{1}, \mathcal{F}_{2}\right):=\sup _{E_{1} \in \mathcal{F}_{1}, E_{2} \in \mathcal{F}_{2}}\left|\mathbb{Q}\left(E_{1} \cap E_{2}\right)-\mathbb{Q}\left(E_{1}\right) \cdot \mathbb{Q}\left(E_{2}\right)\right|
$$

The process $X$ is $\alpha$-mixing, if

$$
\alpha(n) \stackrel{n \rightarrow \infty}{\longrightarrow} 0
$$

Proof. (Lemma 2.1) The stochastic differential equation (11) can be re-written as:

$$
Y(t)=y_{0}+\int_{0}^{t} \mu-\frac{v(s)}{2} d s+\int_{0}^{t} \sqrt{v(s)} d Z(s)
$$

Therefore,

$$
\begin{aligned}
Y(i \Delta)-Y((i-1) \Delta) & =\int_{(i-1) \Delta}^{i \Delta} \mu-\frac{v(s)}{2} d s+\int_{(i-1) \Delta}^{i \Delta} \sqrt{v(s)} d Z(s) \\
& =\mu \Delta-\int_{(i-1) \Delta}^{i \Delta} \frac{v(s)}{2} d s+\int_{(i-1) \Delta}^{i \Delta} \sqrt{v(s)} d Z(s)
\end{aligned}
$$

and

$$
\begin{aligned}
\frac{Y(i \Delta)-Y((i-1) \Delta)}{\sqrt{\Delta}} & =\mu \sqrt{\Delta}-\frac{1}{\sqrt{\Delta}} \int_{(i-1) \Delta}^{i \Delta} \frac{v(s)}{2} d s+\frac{1}{\sqrt{\Delta}} \int_{(i-1) \Delta}^{i \Delta} \sqrt{v(s)} d Z(s) \\
& =\left(\mu-\frac{\bar{V}(i)}{2}\right) \cdot \sqrt{\Delta}+\frac{1}{\sqrt{\Delta}} \int_{(i-1) \Delta}^{i \Delta} \sqrt{v(s)} d Z(s)
\end{aligned}
$$

Proof. (Proposition 2.2) The proof of this proposition works along Sections 3 and 4 of Genon-Catalot (2000). According to them, the volatility process $v$ which is characterized by (2) is $\alpha$-mixing if the following conditions hold, as shown in detail in the example in their Section 4.2:

$$
\begin{aligned}
\kappa_{v} & >0 \\
\kappa_{v} v_{\infty} & \geq \frac{\varepsilon_{v}^{2}}{2}
\end{aligned}
$$

If this is the case, due to Proposition 3.2 in Genon-Catalot (2000) (Note 4), $v(t)$ in (2) is also $\alpha$-mixing. It follows that:

$$
\frac{1}{n} \sum_{i=0}^{n-2} f(R(i+1), R(i+2)) \stackrel{n \rightarrow \infty}{\longrightarrow} \mathbb{E}[\mathbb{E}[f(R(1), R(2))]] \quad \text { a.s. }
$$

where $f$ is chosen (Note 5) to be

$$
f(A, B)=A^{p} \cdot B^{q}
$$

In particular $(p, q) \in\{(1,0),(1,1),(2,0),(2,1),(4,0)\}$. Therefore, $\frac{1}{n} \sum_{i=0}^{n-1} R(i+1) \stackrel{\text { a.s. }}{\longrightarrow} \mathbb{E}[R(1)], \frac{1}{n} \sum_{i=0}^{n-2} R(i+1) R(i+2) \stackrel{\text { a.s. }}{\longrightarrow}$ $\mathbb{E}[R(1) R(2)]$, and so on.

$$
\begin{aligned}
\mathbb{E}[R(1)] & =\mathbb{E}[\mathbb{E}[R(1) \mid \bar{V}]] \\
& =\mathbb{E}\left[\sqrt{\Delta} \mu-\frac{1}{2} \sqrt{\Delta V}(1)\right] \\
& =\sqrt{\Delta} \mu-\frac{1}{2} \sqrt{\Delta} \mathbb{E}[\bar{V}(1)]
\end{aligned}
$$




$$
\begin{aligned}
& \mathbb{E}[R(1) R(2)]= \mathbb{E}[\mathbb{E}[R(1) R(2) \mid \bar{V}]] \\
&= \mathbb{E}[\operatorname{Cov}(R(1), R(2) \mid \bar{V})+\mathbb{E}[R(1) \mid \bar{V}] \cdot \mathbb{E}[R(2) \mid \bar{V}]] \\
&= \mathbb{E}\left[0+\left(\sqrt{\Delta} \mu-\frac{1}{2} \sqrt{\Delta V}(1)\right)\left(\sqrt{\Delta} \mu-\frac{1}{2} \sqrt{\Delta V}(2)\right)\right] \\
&= \mathbb{E}\left[\Delta \mu^{2}-\frac{1}{2} \Delta \mu \bar{V}(1)-\frac{1}{2} \Delta \mu \bar{V}(2)+\frac{1}{4} \Delta \bar{V}(1) \bar{V}(2)\right] \\
&= \Delta \mu^{2}-\frac{1}{2} \Delta \mu \mathbb{E}[\bar{V}(1)]-\frac{1}{2} \Delta \mu \mathbb{E}[\bar{V}(2)]+\frac{1}{4} \Delta \mathbb{E}[\bar{V}(1) \bar{V}(2)] \\
&= \Delta \mu^{2}-\Delta \mu \mathbb{E}[\bar{V}(1)]+\frac{1}{4} \Delta \mathbb{E}[\bar{V}(1) \bar{V}(2)] \\
& \mathbb{E}\left[R^{2}(1)\right]= \mathbb{E}\left[\mathbb{E}\left[R^{2}(1) \mid \bar{V}\right]\right] \\
&= \mathbb{E}\left[\operatorname{Var}(R(1) \mid \bar{V})+\mathbb{E}[R(1) \mid \bar{V}]^{2}\right] \\
&= \mathbb{E}\left[\bar{V}(1)+\left(\sqrt{\Delta \mu}-\frac{1}{2} \sqrt{\Delta V}(1)\right)^{2}\right] \\
&= \mathbb{E}\left[\bar{V}(1)+\Delta \mu^{2}-\Delta \mu \bar{V}(1)+\frac{1}{4} \Delta \bar{V}^{2}(1)\right] \\
&= \Delta \mu^{2}-(\Delta \mu-1) \mathbb{E}[\bar{V}(1)]+\frac{1}{4} \Delta \mathbb{E}\left[\bar{V}^{2}(1)\right] \\
&=\left(\Delta \mu^{2}-(\Delta \mu-1) \mathbb{E}[\bar{V}(1)]+\frac{1}{4} \Delta \mathbb{E}\left[\bar{V}^{2}(1)\right]\right) \\
&= \mathbb{E}\left[\mathbb{E}\left[R(1)^{2} R(2) \mid \bar{V}\right]\right] \\
&= \mathbb{E}\left[\operatorname{Cov}\left(R(1)^{2}, R(2) \mid \bar{V}\right)+\mathbb{E}\left[R(1)^{2} \mid \bar{V}\right] \cdot \mathbb{E}[R(2) \mid \bar{V}]\right] \\
&= \mathbb{E}\left[\mathbb{E}\left[R(1)^{2} \mid \bar{V}\right]\right] \cdot \mathbb{E}[\mathbb{E}[R(2) \mid \bar{V}]] \\
&= \mathbb{E}\left[\mathbb{E}\left[R(1)^{2} \mid \bar{V}\right]\right] \cdot \mathbb{E}[\mathbb{E}[R(1) \mid \bar{V}]] \\
&\left.\mathbb{E}\left[R(1)^{2} R(2)\right]=\frac{1}{2} \sqrt{\Delta} \mathbb{E}[\bar{V}(1)]\right) \\
&= \\
&=
\end{aligned}
$$

For the derivation of $\mathbb{E}\left[R^{4}(1)\right]$ it is used that if $X$ is normally distributed, then the fourth moment of $X$ is

$$
\begin{aligned}
& \mathbb{E}\left[X^{4}\right]=\mathbb{E}[X]^{4}+6 \cdot \mathbb{E}[X]^{2} \cdot \operatorname{Var}(X)+3 \cdot \operatorname{Var}(X)^{2} \\
\mathbb{E}\left[R^{4}(1)\right]= & \mathbb{E}\left[\left[\mathbb{E}\left[R^{4}(1) \mid \bar{V}\right]\right]\right. \\
= & \mathbb{E}[\underbrace{\mathbb{E}[R(1) \bar{V}]^{4}}_{\text {(I) }}+\underbrace{6 \cdot \mathbb{E}[R(1) \mid \bar{V}]^{2} \cdot \operatorname{Var}(R(1) \mid \bar{V})}_{\text {(II) }}+\underbrace{3 \cdot \operatorname{Var}(R(1) \bar{V})^{2}}_{\text {(III) }}] \\
= & \mathbb{E}[\underbrace{\left(\Delta \mu^{2}-\Delta \mu \bar{V}(1)+\frac{1}{4} \Delta \bar{V}^{2}(1)\right)^{2}}_{\text {(I) }} \\
+ & \underbrace{6\left(\Delta \mu^{2}-\Delta \mu \bar{V}(1)+\frac{1}{4} \Delta \bar{V}^{2}(1)\right) \bar{V}(1)}_{\text {(II) }}+\underbrace{3 \bar{V}^{2}(1)}_{\text {(III) }}]
\end{aligned}
$$




$$
\begin{aligned}
= & \mathbb{E}[\underbrace{\Delta^{2} \mu^{4}+\Delta^{2} \mu^{2} \bar{V}^{2}(1)+\frac{1}{16} \Delta^{2} \bar{V}^{4}(1)-2 \Delta^{2} \mu^{3} \bar{V}(1)+\frac{1}{2} \Delta^{2} \mu^{2} \bar{V}^{2}(1)}_{\text {(I) }} \\
\approx & \underbrace{-\frac{1}{2} \Delta^{2} \mu \bar{V}^{3}(1)}_{\text {(I) }}+\underbrace{6 \Delta \mu^{4}+\left(6 \Delta \mu^{2}-2 \Delta^{2}(1)-6 \Delta \mu \bar{V}^{2}(1)+\frac{6}{4} \Delta \bar{V}^{3}\right) \mathbb{E}[\bar{V}(1)]}_{\text {(II) }}+\underbrace{3 \bar{V}^{2}(1)}_{\text {(III) }}] \\
& +\left(\frac{3}{2} \Delta^{2} \mu^{2}-6 \Delta \mu+3\right) \mathbb{E}\left[\bar{V}^{2}(1)\right]
\end{aligned}
$$

Note that the influence of those terms containing $\mathbb{E}\left[\bar{V}^{3}(1)\right]$ or $\mathbb{E}\left[\bar{V}^{4}(1)\right]$ is negligible. Summing up, (33), (34), (35), (36) and (38) prove (16), (17), (18), (19) and (20).

(21), (22) and (23) have already been calculated in Genon-Catalot (2000).

\section{References}

Black, F., \& Cox, J. (1976). Valuing Corporate Securities: Some Effects of Bond Indenture Provisions. Journal of Finance, 31(2), 351-367. http://dx.doi.org/10.2307/2326607

Bollerslev, T. (1986). Generalized Autoregressive Conditional Heteroskedasticity. Journal of Econometrics, 31, 307-327. http://dx.doi.org/10.1016/0304-4076(86)90063-1

Bradley, R. (2005). Basic Properties of Strong Mixing Conditions. A Survey and Some Open Questions. Probability Surveys, 2, 107-144.

Brockwell, P., Chadraa, E., \& Lindner, A. (2006). Continuous time GARCH processes. The Annals of Applied Probability, 16(2), 790-826. http://dx.doi.org/10.1214/105051606000000150

Chernoff, H., \& Lehmann, E. (1954). The Use of Maximum Likelihood Estimates in Chi-Square Tests for Goodness of Fit. The Annals of Mathematical Statistics, 25(3), 579-586. http://dx.doi.org/10.1214/aoms/1177728726

Cox, J., Ingersoll, J., \& Ross, S. (1985). A Theory of the Term Structure of Interest Rates. Econometrica, 2, 385-408. http://dx.doi.org/10.2307/1911242

Davidson, R., \& MacKinnon, J. (1993). Estimation and Inference in Econometrics. Oxford University Press.

Embrechts, P., Frey, R., \& McNeil, A. (2005). Quantitative Risk Management. Princeton University Press.

Engle, R. (1982). Autoregressive Contidional Heteroscedasticity with Estimates of the Variance of United Kingdom Inflation. Econometrica, 50(4), 987-1008. http://dx.doi.org/10.2307/1912773

Escobar, M., Friederich, T., Seco, L., \& Zagst, R. (2011). A General Structural Approach for Credit Modeling under Stochastic Volatility. Journal of Financial Transformation, 32, 123-132.

Feller, W. (1951). Two Singular Diffusion Problems. The Annals of Mathematics, 54(1), 173-182. http://dx.doi.org/10.2307/1969318

Genon-Catalot, V., Jeantheau, T., \& Laredo, C. (2000). Stochastic Volatility Models as Hidden Markov Models and Statistical Applications. Bernoulli, 60(6), 1051-1079. http://dx.doi.org/10.2307/3318471

Heston, S. (1993). A Closed-Form Solution for Options with Stochastic Volatility with Applications to Bond and Currency Options. The Review of Financial Studies, 6(2), 327-343. http://dx.doi.org/10.1093/rfs/6.2.327

Merton, R. (1973). Theory of Rational Option Pricing. Bell Journal of Economics and Management Science, 4, 141-183. http://dx.doi.org/10.2307/3003143

Rosenblatt, M. (1956). A central limit theorem and a strong mixing condition. Proceedings of the National Academy of Sciences, 42, 43-47. http://dx.doi.org/10.1073/pnas.42.1.43

Zagst, R. (2002). Interest Rate Models. Springer Finance. 
Table 1. Numerical and analytical pricing of a DOC in the stochastic volatility model

\begin{tabular}{rrrrrrrr}
\hline zero & $\begin{array}{r}C_{\text {num }} \\
\text { payoffs }\end{array}$ & $\begin{array}{c}C \\
\text { mean }\end{array}$ & analytical & diff. & sigma & \multicolumn{2}{c}{ 95\% confidence } \\
lower & upper \\
\hline 0.00 & 0 & 99.936 & 100.000 & -0.064 & 22.507 & 99.494 & 100.377 \\
10.00 & 0 & 89.877 & 90.000 & -0.123 & 22.749 & 89.431 & 90.323 \\
20.00 & 0 & 79.845 & 80.000 & -0.155 & 22.624 & 79.402 & 80.288 \\
30.00 & 0 & 70.118 & 70.000 & 0.118 & 22.864 & 69.670 & 70.566 \\
40.00 & 0 & 60.350 & 60.000 & 0.350 & 22.649 & 59.906 & 60.794 \\
50.00 & 14 & 49.776 & 50.000 & -0.224 & 22.026 & 49.345 & 50.208 \\
60.00 & 288 & 40.240 & 40.000 & 0.240 & 22.888 & 39.792 & 40.689 \\
70.00 & 1291 & 29.737 & 30.000 & -0.263 & 22.098 & 29.304 & 30.170 \\
80.00 & 3479 & 20.163 & 20.000 & 0.163 & 21.617 & 19.739 & 20.587 \\
90.00 & 6637 & 10.003 & 10.000 & 0.003 & 17.764 & 9.655 & 10.352 \\
\hline
\end{tabular}

Table 2. Consolidated financial data of Merrill Lynch 2000-2008

\begin{tabular}{lrrrrrrrrr}
\hline *(in million USD) & $\mathbf{2 0 0 0}$ & $\mathbf{2 0 0 1}$ & $\mathbf{2 0 0 2}$ & $\mathbf{2 0 0 3}$ & $\mathbf{2 0 0 4}$ & $\mathbf{2 0 0 5}$ & $\mathbf{2 0 0 6}$ & $\mathbf{2 0 0 7}$ & $\mathbf{2 0 0 8}$ \\
\hline EBIT* & 5717 & 1377 & 3757 & 5649 & 5836 & 7231 & 10426 & -12831 & -41831 \\
Net Revenues* & 26766 & 21880 & 18608 & 20154 & 22023 & 26009 & 34659 & 11250 & -12593 \\
Total Assets* & 407200 & 419419 & 447928 & 494518 & 648059 & 681015 & 841299 & 1020050 & 667543 \\
Total Liabilities* & 386182 & 396716 & 422395 & 464197 & 616689 & 645415 & 802261 & 988118 & 647540 \\
Stockholders' Equity* & 18304 & 20008 & 22875 & 27651 & 31370 & 35600 & 39038 & 31932 & 20003 \\
Adjusted Leverage & 13.2 & 13.1 & 11.1 & 11.7 & 13.9 & 11.6 & 13.1 & 17.7 & 13.3 \\
\hline
\end{tabular}
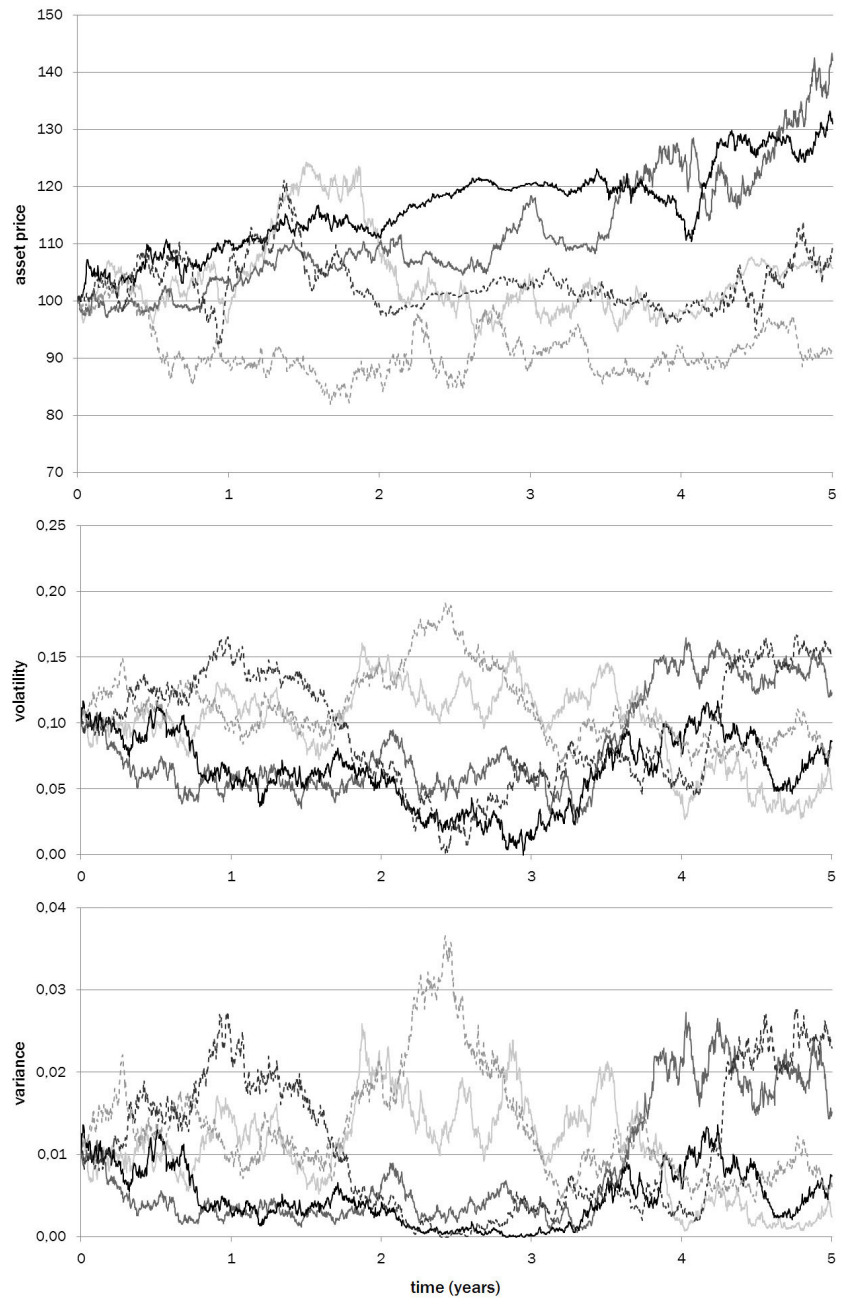

Figure 1. Five simulated paths in the stochastic volatility model 


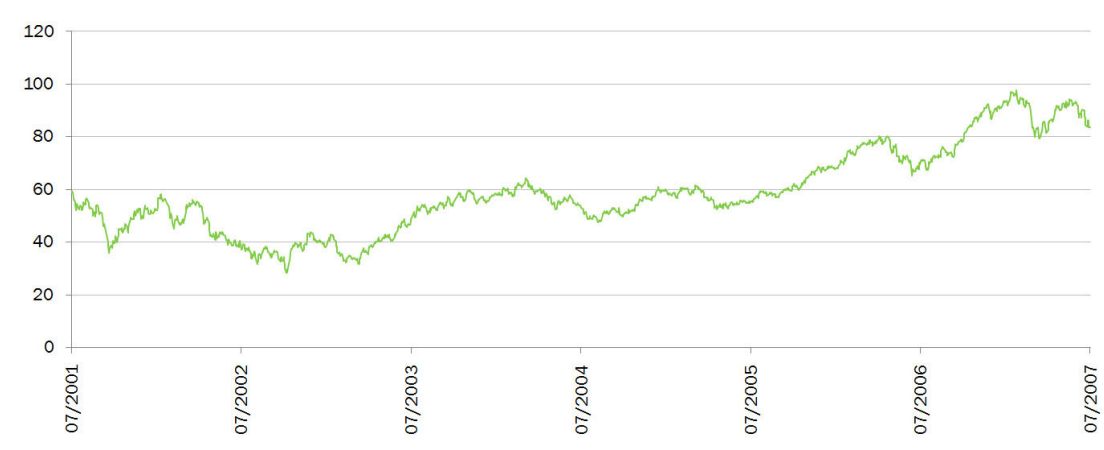

Figure 2. Performance of Merrill Lynch Stocks on NYSE (July 2001 - June 2007)

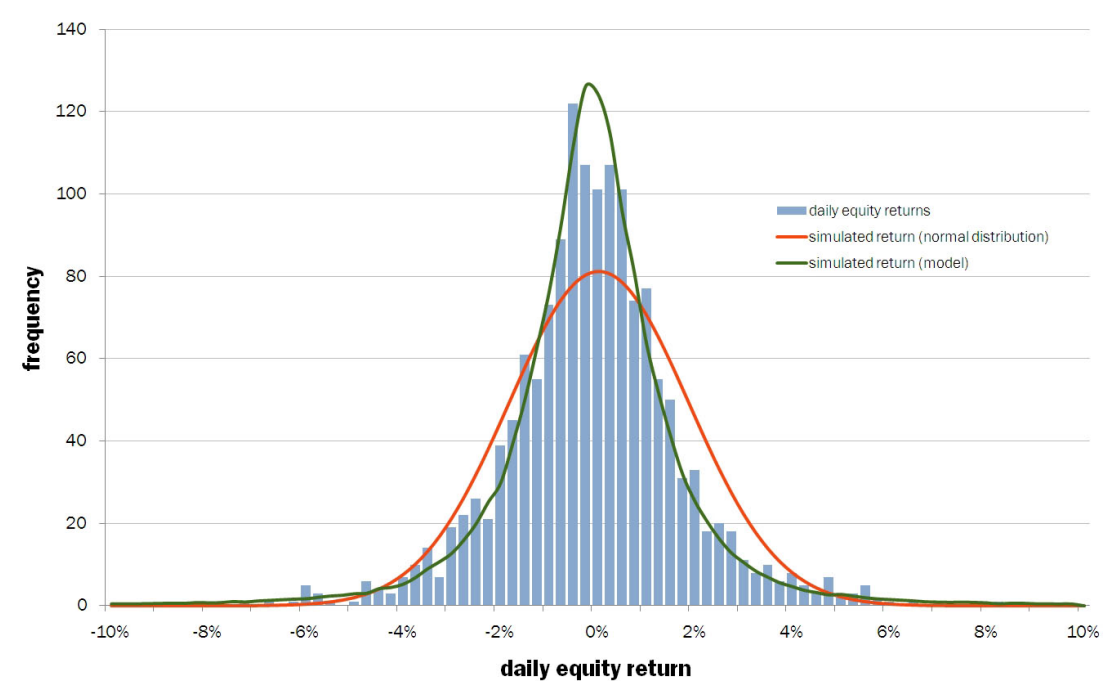

Figure 3. Observed equity returns of Merrill Lynch (July 2001 - June 2007) and simulated returns

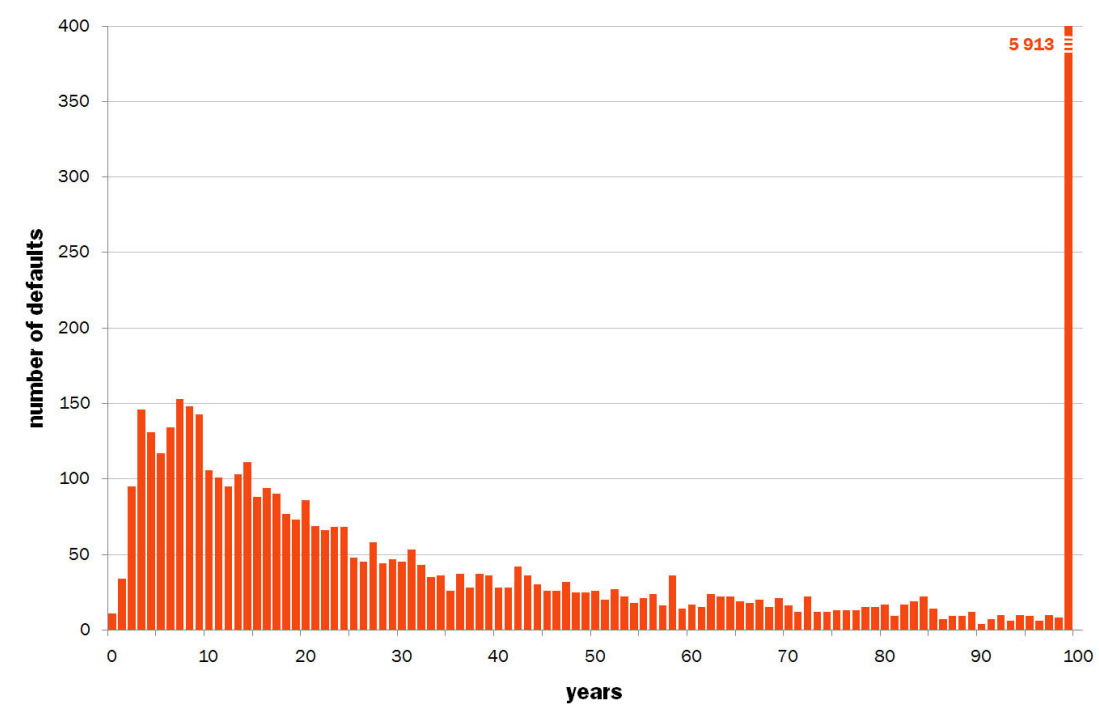

Figure 4. Number of defaults out of 10000 simulated time series for Merrill Lynch (July 2007) 


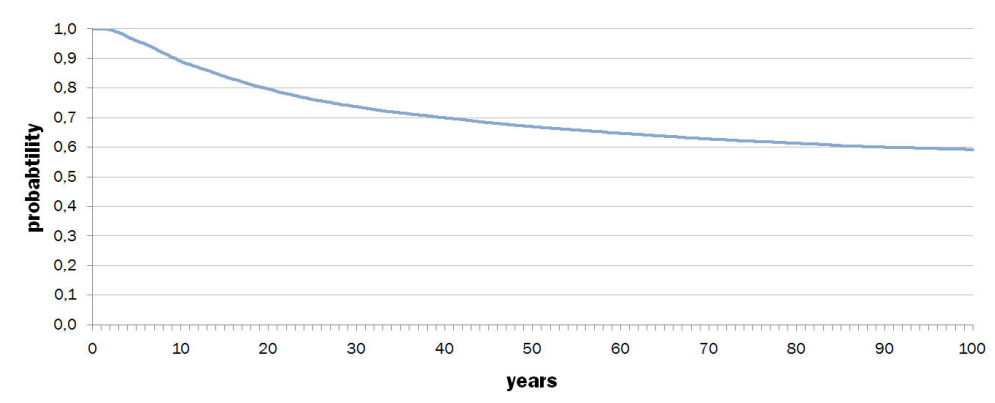

Figure 5. Survival probability for Merrill Lynch (July 2007)

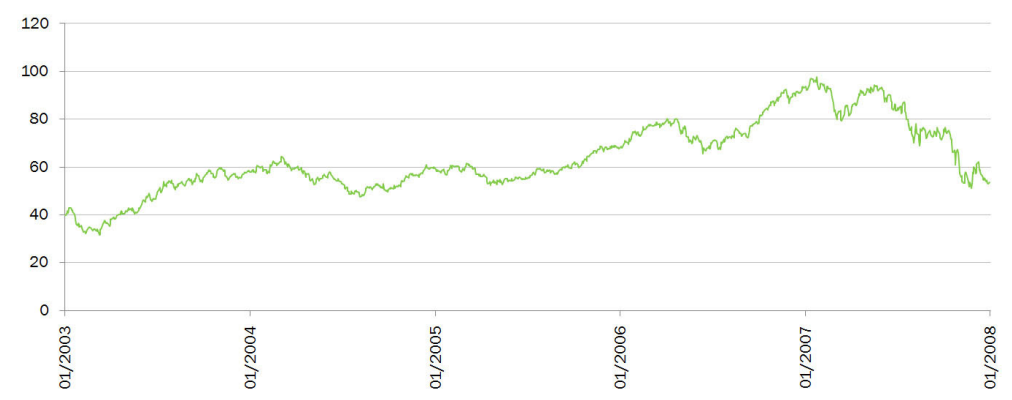

Figure 6. Performance of Merrill Lynch Stocks on NYSE (January 2003 - December 2007)

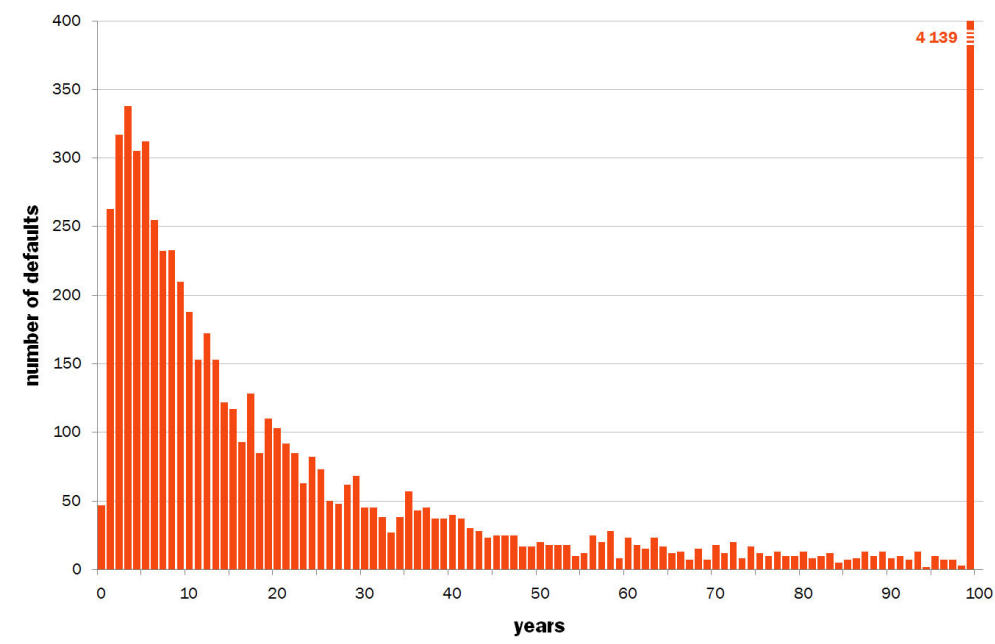

Figure 7. Number of defaults out of 10000 simulated time series for Merrill Lynch (January 2008)

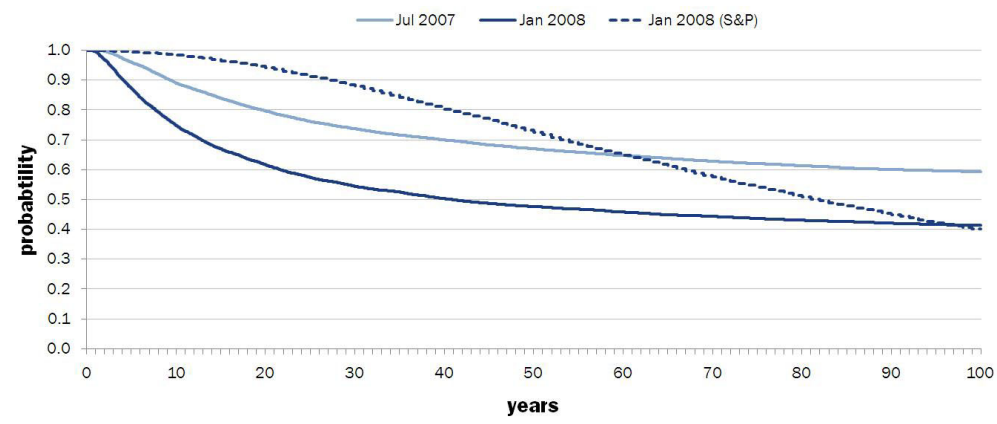

Figure 8. Survival probability for Merrill Lynch (July 2007 and January 2008) 
Notes

Note 1. The basic idea of a barrier option is that the option expires if the underlying hits a barrier ("out options") or becomes exercisable only if such a barrier is reached ("in options"). A down-and-out call option therefore describes a call option which expires if the underlying falls below a certain barrier. Thus, barrier options are path-dependent.

Note 2. In order to accurately fit the parameters of the volatility process which is not directly observable, we need sufficiently many data points. Thus, daily data is used in the example.

Note 3. The adjusted leverage ratio is defined in the annual reports as "assets reduced by securities financing transactions and securities received as collateral less trading liabilities net of derivative contracts and segregated cash and securities and separate accounts assets, [...] divided by equity capital."

Note 4. Although it is not stated in the proof of that proposition, it may be noted that the basic for this finding has already been laid by Rosenblatt (1956).

Note 5. The Proposition in Genon-Catalot (2000) deals with a more general class of functions. 